\title{
Mobilitätshemmnisse heterogener Arbeitskräfte in Deutschland
}

\author{
Melanie Arntz
}

Angenommen: 21. Dezember 2010 / Online publiziert: 15. Februar 2011

(C) Institut für Arbeitsmarkt- und Berufsforschung 2011

Zusammenfassung Die Binnenmigration fällt im internationalen Vergleich in Deutschland relativ schwach aus. Die räumliche Mobilität der Arbeitskräfte kann jedoch einen wichtigen Beitrag zum Wirtschaftswachstum und zum Ausgleich interregionaler Disparitäten leisten, so dass die Identifikation von Mobilitätshemmnissen von hoher wirtschaftspolitischer Relevanz ist. Ziel des in diesem Artikel diskutierten Forschungsprojektes ist es, institutionelle Ursachen einer niedrigen Mobilitätsbereitschaft von Arbeitslosen nachzugehen und dabei auch die Frage zu untersuchen, ob institutionelle Faktoren wie z.B. die aktive und passive Arbeitsmarktpolitik eine Teilerklärung für die große Mobilitätslücke zwischen qualifizierten und unqualifizierten Arbeitskräften in Deutschland liefern. Die Ergebnisse deuten darauf hin, dass eine generöse Arbeitslosenunterstützung die Mobilitätsbereitschaft senkt. Workfare-Programme, die zu einer Absenkung der teilweise hohen Lohnersatzquoten von Geringqualifizierten beitragen, könnten somit zu einer erhöhten Binnenmigration beitragen.

\section{Barriers to the mobility of heterogeneous labour in Germany}

Abstract Internal migration rates in Germany are relatively low in international comparison. Identifying obstacles to interregional mobility is thus of major policy concern because the geographic mobility of labour may contribute to higher

J. Prof. Dr. M. Arntz $(\bowtie)$

Centre for European Economic Research (ZEW), L7 1,

68161 Mannheim, Deutschland

e-mail: arntz@zew.de

J. Prof. Dr. M. Arntz

University of Heidelberg, Bergheimerstr. 58, 69115 Heidelberg,

Deutschland overall economic growth as well as a reduction of interregional employment disparities. This article presents a number of findings from a research project that aims at identifying institutional factors that reduce geographic mobility among unemployed jobseekers in Germany and that, at the same time, may also contribute to explaining the large gap in internal migration rates between qualified and unqualified workers. The findings indicate that a generous unemployment benefit system contributes to the observed phenomena. Workfare programs that reduce the partially high wage replacement rates among low-qualified unemployed might thus result in higher levels of internal migration in Germany.

\section{Einleitung}

In einer Studie der OECD (2005) zur Binnenmigration ${ }^{1}$ in verschiedenen Ländern bestätigt sich eine vielfach gemachte Beobachtung: interne Migrationsraten fallen in europäischen Ländern niedriger aus als in den USA oder Australien, wobei Ost- und Südeuropa die niedrigsten Migrationsraten und Westeuropa, allen voran Großbritannien, die höchsten Migrationsraten aufweist. Deutschland rangiert im innereuropäischen Vergleich in der Mitte. Ähnliche ländervergleichende Ergebnisse finden sich auch in Faini (1999) und Braunerhjelm et al. (2000).

Ein besseres Verständnis der Ursachen dieser beobachteten Unterschiede ist von hoher politischer Relevanz, da die räumliche Mobilität von Arbeitskräften, sei es durch interregionale Umzüge oder die Bereitschaft zum interregionalen Pendeln, einen nicht unwesentlichen Beitrag zu einem

\footnotetext{
${ }^{1}$ Binnenmigration, interregionale Mobilität und räumliche Mobilität werden synonym verwendet und beziehen sich stets auf einen Umzug innerhalb eines Landes über regionale Grenzen hinweg.
} 
Abb. 1 Anteil an Personen zwischen 15-64 Jahren, die in 2003 ihren Wohnort überregional verlegt haben, Quelle: Organization for Economic Co-operation and Development. OECD Employment Outlook (2005), S. 94

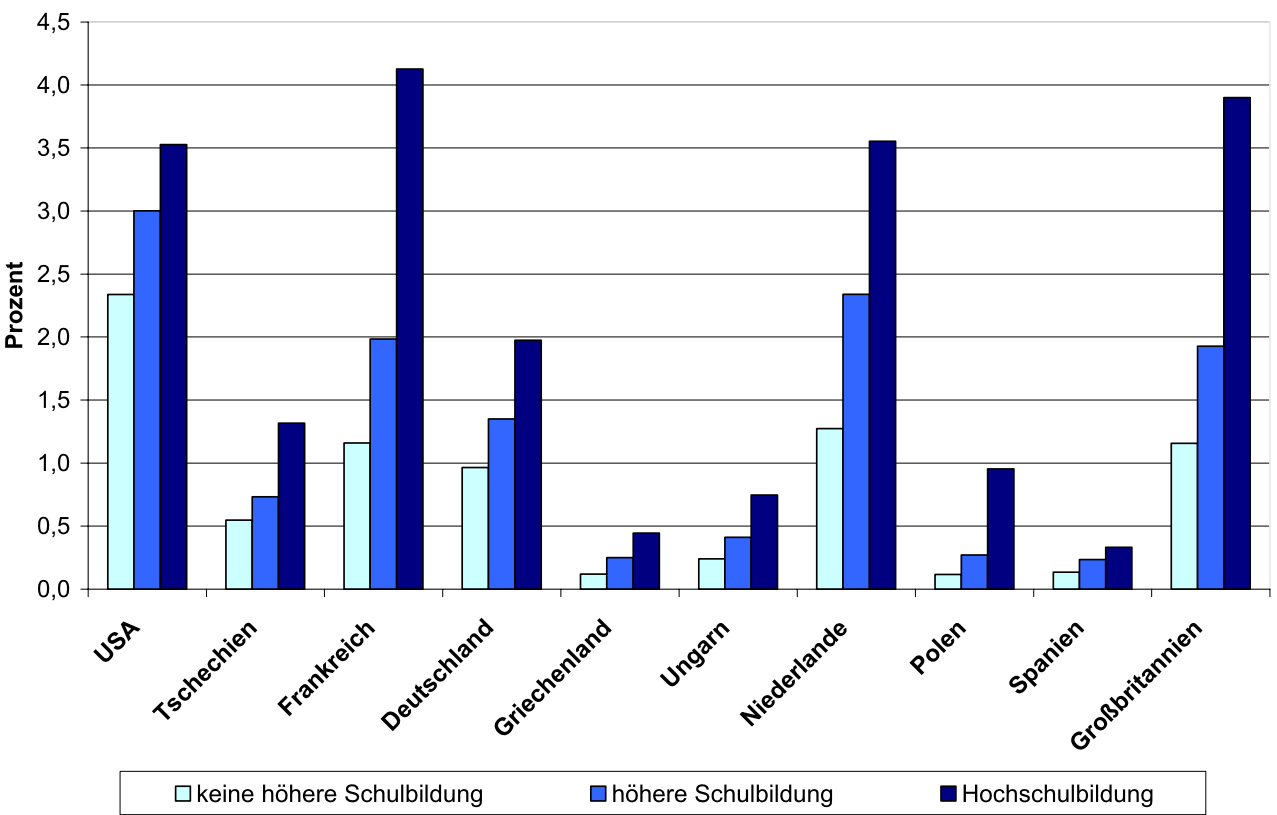

Anmerkung: Ein überregionaler Umzug entspricht in den USA einem Umzug in einen anderen Bundesstaat, in Europa entspricht er einem Umzug in eine andere NUTS2 Region. höheren Wachstumspfad leisten kann. Ein höheres Wachstum kann insbesondere durch den Abbau eines räumlichen Mismatches zwischen Arbeitsangebot und Arbeitsnachfrage und damit höheren Beschäftigungsraten gelingen. Darüber hinaus kann die räumliche Mobilität der Arbeitskräfte dazu beitragen, interregionale Beschäftigungs- und Einkommensdisparitäten abzubauen. Idealtypisch führt ein negativer regionaler Arbeitsnachfrageschock zu einer steigenden Arbeitslosigkeit bei gleichzeitig sinkenden Löhnen sowie einer sinkenden Partizipation am Arbeitsmarkt. Die niedrigen Löhne stimulieren einerseits die Arbeitsnachfrage, andererseits verursachen sie eine Nettoabwanderung. In der Folge dieser Anpassungsprozesse erreichen die Partizipationsund Arbeitslosenquote ebenso wie die Löhne das ursprüngliche Niveau. Für die USA, Australien und Neuseeland konnten Studien nachweisen, dass eine hohe Abwanderungsneigung im Zuge eines regionalen Schocks ein wesentlicher Bestandteil dieses Anpassungsmechanismus ist (vgl. Blanchard und Katz 1992; Debelle und Vickery 1999; Choy et al. 2002). Demgegenüber fällt die Anpassung durch Abwanderungsprozesse in einem europäischen Kontext sehr schwach aus, so dass in der Folge eines regionalen Arbeitsnachfrageschocks primär die Partizipationsraten reagieren (vgl. Decressin und Fatàs 1995; Nahuis und Parikh 2002). Für Deutschland konnte Möller (1995) zwar nachweisen, dass die Binnenmigration langfristig einen wichtigen Beitrag zum regionalen Anpassungsprozess leistet, dies in der kurzen Frist jedoch kaum wirksam ist.

Die Identifikation von Mobilitätshemmnissen ist daher von hoher politischer Relevanz. Allerdings deutet die Bin- nenmigration verschiedener Bildungsgruppen in Abb. 1 darauf hin, dass dieses Vorhaben die Heterogenität verschiedener Arbeitsmarktgruppen berücksichtigen muss. Insbesondere zeigt sich die in allen Ländern niedrigere Mobilität unqualifizierter Arbeitskräfte im Vergleich zu hochqualifizierten Arbeitskräften.

Dies deutet darauf hin, dass das Mobilitätskalkül, d.h. der Vergleich des erwarteten zukünftigen Nutzens in einer potenziellen Zielregion abzüglich der Migrationskosten mit dem erwarteten Nutzen des Verbleibs in der Herkunftsregion, individuell unterschiedlich ausfällt, wobei Migrationskosten monetäre und nicht-monetäre Kosten der Mobilität umfassen und somit neben den direkten Umzugskosten auch psychologische Kosten und Informationskosten mit einschließen (vgl. Sjaastad 1962; Shields und Shields 1989). Die geringere Mobilitätsneigung unqualifizierter Arbeitskräfte deutet darauf hin, dass diese im Vergleich zu qualifizierten Arbeitskräften insgesamt einen geringeren Nutzenzuwachs durch Migration nach Abzug der Migrationskosten erzielen. $^{2}$ Die größere Mobilitätslücke zwischen unqualifizierten und qualifizierten Arbeitskräften in Europa im Vergleich zu den USA (vgl. Abb. 1) weist zudem darauf hin, dass institutionelle Gegebenheiten zu Mobilitätshemmnis-

\footnotetext{
${ }^{2}$ Während Hochqualifizierte vermutlich häufiger von hohen monetären Migrationskosten beispielsweise aufgrund von Wohneigentum betroffen sind, sind Geringqualifizierte möglicherweise von höheren Informationskosten sowie psychologischen Kosten betroffen, da sie über weniger überregionale soziale Netzwerke verfügen.
} 
sen beitragen, die unqualifizierte Arbeitskräfte in stärkerem Maße betreffen.

Institutionelle Rahmenbedingungen, die sich zwischen den USA und Deutschland unterscheiden und zumindest eine potenzielle Teilerklärung für die in Deutschland größere Mobilitätslücke zwischen Unqualifizierten und Qualifizierten liefern, sind beispielweise die großzügigeren Regelungen zur aktiven und passiven Arbeitsmarktpolitik. Ein wesentliches Ziel des DFG-Forschungsprojektes „Flexibilisierungspotenziale heterogener regionaler Arbeitsmärkte durch räumliche Mobilität“政 war es, institutionelle Mobilitätsbarrieren in Deutschland zu identifizieren und dabei ein besonderes Augenmerk auf das Mobilitätsverhalten unterschiedlicher Qualifikationsgruppen zu legen. Ein Teil der im Rahmen des Projektes gewonnenen Erkenntnisse werden im Folgenden anhand dreier Forschungsarbeiten erläutert, deren wirtschaftspolitische Relevanz im Anschluss diskutiert wird.

\section{Erstes Ergebnis: Unqualifizierte Arbeitslose reagieren kaum auf regionale Arbeitsmarktbedingungen}

Bisherige Studien für Deutschland konnten zwar wiederholt zeigen, dass die Elastizität von Migrationsströmen im Hinblick auf interregionale Lohn- und Beschäftigungsunterschiede in Deutschland im Verhältnis zu den USA relativ schwach ausfallen (vgl. Puhani 2001); die unterschiedliche Mobilitätsneigung verschiedener Qualifikationsgruppen war bislang jedoch kein Kernthema der Forschung. Im Vordergrund des Forschungsprojektes stand daher zunächst die Frage, ob und in welchem Maße Individuen unterschiedlichen Qualifikationsniveaus auf ungünstige regionale $\mathrm{Ar}$ beitsmarktbedingungen mit Abwanderung reagieren. Dabei lag der Fokus auf Jobsuchenden, da deren Mobilitätsbereitschaft ein entscheidender Anpassungsmechanismus zum Abbau regionaler Beschäftigungsdisparitäten ist. Die Analyse in Arntz (2005) unterscheidet daher zwischen unqualifizierten und qualifizierten Arbeitslosen, wobei letztere entweder eine Lehre oder ein Studium abgeschlossen haben.

Als theoretische Grundlage der Analyse dient ein räumliches Suchmodell, welches davon ausgeht, dass Arbeitslose unter Unsicherheit simultan ${ }^{4}$ einen Job im regionalen und im überregionalen Arbeitsmarkt suchen (vgl. Herzog et al.

\footnotetext{
${ }^{3}$ Das Projekt wurde von 2004 bis 2008 von Prof. H. Entorf (derzeit: Johann Wolfgang Goethe-Universität Frankfurt) und seit 2008 von J. Prof. Dr. M. Arntz (ZEW, Mannheim und Ruprecht-KarlsUniversität Heidelberg) geleitet.

${ }^{4}$ Eine Jobsuche kann alternativ auch sequentiell erfolgen (McCall und McCall 1987).
}

1993; Damm und Rosholm 2003). ${ }^{5}$ Die Wahrscheinlichkeit einer Beschäftigung in einem der Märkte setzt sich nun zusammen aus der Wahrscheinlichkeit, dort ein Jobangebot zu erhalten und der Wahrscheinlichkeit, dieses zu akzeptieren. Diese Wahrscheinlichkeiten hängen jedoch nicht nur von exogenen Gegebenheiten der Arbeitsmärkte ab, sondern auch von der endogenen Suchstrategie des Arbeitsuchenden. Diese besteht zum einen aus den Reservationslöhnen, deren Höhe so gesetzt wird, dass der Wert einer Beschäftigung zu diesem Lohnsatz in der jeweiligen Region gerade dem Wert einer fortgesetzten Arbeitslosigkeit entspricht. Zum anderen alloziert der Arbeitssuchende seine Suchanstrengungen derart, dass der marginale Wert der Suchanstrengung in einer Region gerade den marginalen Kosten dieser Suchanstrengung entspricht. Exogen verbesserte Arbeitsmarktbedingungen in einer anderen Region relativ zur Heimatregion erhöhen nach dieser Theorie den Reservationslohn für alle regionalen Arbeitsmärkte, führen jedoch zu einer Verlagerung der Suchanstrengung zugunsten des nun attraktiveren Teilmarktes.

Vor dem Hintergrund dieser theoretischen Zusammenhänge untersucht die empirische Analyse, in welchem MaBe unqualifizierte und qualifizierte Arbeitslose mit einer erhöhten Abwanderungsneigung reagieren, wenn sich Arbeitsbedingungen im überregionalen Arbeitsmarkt relativ zum regionalen Arbeitsmarkt verbessern. Die Analyse beruht auf administrativen Mikrodaten, der regionalen Version der IAB ${ }^{6}$ Beschäftigtenstichprobe. Dieser Datensatz repräsentiert $1 \%$ der sozialversicherungspflichtigen Erwerbstätigen, für welche die Beschäftigungshistorie tagesgenau festgehalten und anhand der Betriebsstätten lokalisiert ist (Bender et al. 2000). Die empirische Analyse beschränkt sich auf westdeutsche Männer, die zwischen 1983 und 1997 arbeitslos waren. Getrennt für unqualifizierte und qualifizierte Arbeitslose identifiziert die Analyse die Determinanten der Verweildauer in Arbeitslosigkeit bis zu einem Übergang in eine regionale oder überregionale Beschäftigung.

Die Resultate für qualifizierte Arbeitslose zeigen, dass die Wahrscheinlichkeit einer überregionalen Beschäftigung nach einem Jahr um $13 \%$ steigt, wenn sich die regionalen Beschäftigungschancen im Vergleich zu anderen Regionen verschlechtern. Demgegenüber verharren unqualifizierte Arbeitslose auch bei sich regional verschlechternden Beschäftigungschancen in der Region. Die Ergebnisse bestätigen daher eine geringere räumliche Mobilität von unqualifizierten im Vergleich zu qualifizierten Arbeitslosen. Die sich anschließenden Analysen des Forschungsprojektes widmen sich nun der Frage, welche institutionellen Faktoren, diese unterschiedliche Mobilitätsneigung erklären können.

\footnotetext{
${ }^{5}$ Dieses Modell ist übertragbar auf eine interindustrielle (vgl. Fallick 1992) oder intersektorale Suche (vgl. Thomas 1998).

${ }^{6}$ Institut für Arbeitsmarkt- und Berufsforschung, Nürnberg.
} 


\section{Zweites Ergebnis: Die aktive Arbeitsmarktpolitik ist kein besonderes Mobilitätshemmnis für einkommensschwache Arbeitslose}

Die Teilnahme an einem aktiven Arbeitsmarktprogramm (AAMP), wie z.B. eine subventionierte Beschäftigung im primären oder im sekundären Arbeitsmarkt, eine Arbeitsbeschaffungsmaßnahme oder eine subventionierte Selbstständigkeit, kann die Kosten einer verlängerten Arbeitslosigkeit reduzieren. Das regionale Angebot an solchen Programmen verändert daher möglicherweise das Suchkalkül des Arbeitssuchenden. In einem um einen regional organisierten, subventionierten Arbeitsmarkt erweiterten interregionalen Suchmodell wird die Aufnahme einer subventionierten Beschäftigung durch ein umfangreiches Angebot an AAMP begünstigt, indem dies zu verstärkten Suchanstrengungen in diesem Teilmarkt auf Kosten der Suchanstrengungen im regulären und vor allem überregionalen Arbeitsmarkt führt. Tatsächlich konnten Frederiksson und Johansson (2003) und Lindgren und Westerlund (2003) für Schweden zeigen, dass die Teilnahme an AAMP die räumliche Mobilität senkt. Zudem reduzierte ein umfangreiches lokales Angebot an AAMP die Bruttoabwanderungsraten in Schweden (vgl. Westerlund 1997, 1998). Möglicherweise ist ein mobilitätshemmender Effekt jedoch vor allem für die Arbeitskräfte wirksam, für die eine solche Maßnahme aufgrund eines niedrigen Bildungsniveaus ein attraktives Substitut für eine reguläre Beschäftigung darstellt.

Um diese Hypothese zu testen, untersucht die zweite Studie des Forschungsprojektes (Arntz und Wilke 2009) für männliche Arbeitslose in Westdeutschland den Einfluss des lokalen AAMP Angebotes auf die Verweildauer in Arbeitslosigkeit bis zu einem Übergang in eine der drei konkurrierenden Beschäftigungsmöglichkeiten (regional, überregional, AAMP). Dies ist möglich auf Basis der Integrierten Erwerbsbiographien V.1 des IAB, die etwa 2,2 \% der sozialversicherungspflichtigen Beschäftigten enthalten (vgl. Jacobebbinghaus und Seth 2007) und für den Zeitraum von 2000-2004 zusätzlich zu den Übergängen von der Arbeitslosigkeit in eine regionale oder eine überregionale Beschäftigung auch Übergänge in eine AAMP beinhalten.

Die nach Einkommensgruppen getrennt durchgeführten Analysen zeigen, dass eine Ausdehnung des lokalen Angebotes an AAMP die Übergänge in eine subventionierte Beschäftigung zu Ungunsten der Übergänge in eine reguläre Beschäftigung beschleunigen. Nur bei unverheirateten Arbeitslosen schlägt sich die reduzierte Wahrscheinlichkeit einer regulären Beschäftigung auch in einer signifikant reduzierten Wahrscheinlichkeit einer überregionalen Beschäftigung nieder. Für Verheiratete mit vermutlich höheren Mobilitätskosten haben die regionalen Arbeitsmarktbedingungen somit zwar einen Einfluss auf die Arbeitslosigkeitsdauer, doch an der Bereitschaft, überregional eine Beschäftigung aufzunehmen, ändert sich wenig. Der mobilitätshemmende Effekt des regionalen Angebots an AAMP auf Unverheiratete fällt zudem für einkommensschwache Einkommensgruppen deutlich abgeschwächt aus.

Es zeigt sich somit zwar, dass ein umfangreiches Angebot an AAMP auf Arbeitslose mit niedrigen Mobilitätskosten einen leicht mobilitätshemmenden Einfluss ausübt. Das regionale Angebot an AAMP kann jedoch nicht die Mobilitätslücke zwischen einkommensschwachen und somit zumeist weniger qualifizierten Arbeitskräften und einkommensstarken, qualifizierten Arbeitskräften erklären. In einem nächsten Forschungsschritt wendet sich das Forschungsprojekt daher dem Einfluss der Arbeitslosenunterstützung zu.

\section{Drittes Ergebnis: Eine großzügige Arbeitslosenunterstützung reduziert die Mobilitätsbereitschaft}

Während Arbeitslose im Vergleich zu Beschäftigten üblicherweise eine höhere Mobilität aufweisen (vgl. Herzog und Schlottmann 1984; Pissarides und Wadsworth 1989; Jackman und Savouri 1992), spricht die Suchtheorie dafür, dass die Arbeitslosenunterstützung mobilitätshemmend wirkt, da diese die Kosten einer verlängerten Arbeitslosigkeit in der Region reduziert (Hassler et al. 2005). Insbesondere sollte die Mobilitätsbereitschaft mit der Lohnersatzquote, d.h. dem in Prozent des vorherigen Lohns erhaltene Lohnersatz, sinken. Da einkommensschwache Arbeitslose in Deutschland aufgrund aufstockender Sozialhilfe ${ }^{7}$ höhere Lohnersatzquoten erzielen als qualifizierte und einkommensstärkere Arbeitskräfte, sind Mobilitätsanreize für die erstgenannte Gruppe möglicherweise besonders stark herabgesetzt.

In den vorhandenen administrativen Mikrodaten fehlen jedoch Informationen zur Berechnung der Lohnersatzquoten. Da der Umfang der Arbeitslosenunterstützung jedoch nicht allein von der Lohnersatzquote, sondern auch von der maximalen Anspruchsdauer auf Arbeitslosengeld (ALG) abhängt und diese Anspruchsdauern auf Basis der beobachtbaren Erwerbshistorie berechnet werden können, untersucht die im vorherigen Abschnitt skizzierte Forschungsarbeit (Arntz und Wilke 2009) neben den Wirkungen des regionalen Angebots an AAMP auch den Einfluss der maximalen Bezugsdauer.

Dabei ist jedoch zu berücksichtigen, dass eine Verkürzung/Verlängerung der ALG-Anspruchsdauer keine Einkommensveränderungen nach sich zieht, wenn der Betroffene zusätzlich aufstockende Sozialhilfe bezieht und somit

\footnotetext{
${ }^{7}$ Die Studie wurde mit Daten vor Einführung des Arbeitslosengelds II durchgeführt.
} 
eine Lohnersatzquote erhält, die auch nach Ablauf des Arbeitslosengeldes oberhalb der Lohnersatzquote des Arbeitslosengeldes von $63 \%$ bzw. $68 \%$ liegt. Auch für diejenigen, die zwar keine aufstockende Sozialhilfe erhalten, aber nach Ablauf des Arbeitslosengeldes aufgrund der Einkommenssituation des Haushaltes Arbeitslosenhilfe in der Höhe von 53-57\% des vorherigen Lohns beziehen, ändert eine kürzere Bezugsdauer des Arbeitslosengeldes nur wenig. Lediglich die Gruppe der Arbeitslosen, die nach Ablauf des Arbeitslosengeldes keine weiteren Leistungen erhalten, haben eine nennenswerte Einkommenseinbuße von 63-68\% ihres vorherigen Lohneinkommens zu verzeichnen. Der Einfluss der Arbeitslosenunterstützung sollte sich daher nur für die letztgenannte Gruppe nachweisen lassen. Da die von der ALG-Anspruchsdauer unterschiedlich betroffenen Personen in den Daten nicht exakt identifiziert werden können und wir auch das Ende der ALG-Anspruchsdauer nur bei der selektiven Gruppe der Arbeitslosen beobachten können, die dauerhaft keine Beschäftigung finden, unterscheidet die Analyse stattdessen verschiedene Einkommens- und Bildungsgruppen, die mit einer unterschiedlichen Wahrscheinlichkeit von einer aufstockenden Sozialhilfe betroffen sein sollten. Geringverdiener und Unqualifizierte sollten besonders häufig eine aufstockende Sozialhilfe beziehen, so dass der Einfluss der Arbeitslosenunterstützung für diese Gruppe kaum nachweisbar sein sollte.

Die Schätzergebnisse zeigen wie erwartet, dass die ALGAnspruchsdauer einen stärkeren Einfluss auf die höheren als auf die niedrigeren Lohngruppen hat. Insbesondere hat eine längere ALG-Anspruchsdauer und damit eine großzügigere Arbeitslosenunterstützung für diese Lohngruppen nicht nur den in der Literatur bereits bekannten Effekt, die Dauer der Arbeitslosigkeit zu verlängern, sondern senkt darüber hinaus auch die Mobilitätsbereitschaft der Betroffenen. Problematisch an diesen Ergebnissen ist jedoch, dass die geschätzte Wirkung möglicherweise nicht kausal auf die Anspruchsdauern, sondern auch auf eine unbeobachtete Selektion in die Länge der Anspruchsdauern zurückgeht. Denkbar ist beispielsweise, dass sich Individuen mit einer kurzen Anspruchsdauer nicht nur in beobachtbaren sondern auch aufgrund unbeobachtbarer Merkmale wie z.B. der Motivation von Individuen unterscheiden, die aufgrund einer stabileren Erwerbskarriere längere Anspruchsdauern aufweisen.

Um daher die kausale Wirkung der Arbeitslosenunterstützung besser einzugrenzen, nutzt eine weitere Forschungsarbeit (Arntz et al. 2008) eine quasi-experimentelle Variation in den maximalen Bezugsdauern für das Arbeitslosengeld. So wurden die ALG-Anspruchsdauern für Arbeitslose im Alter von 42-43 Jahren 1997 von 18 auf 12 Monate und für 44-jährige Arbeitslose von 22 auf 12 Monate gesenkt. Ein Vergleich des Verlaufs der Arbeitslosigkeit und der Übergänge in eine regionale oder überregionale Beschäftigung dieser von einer Kürzung der Anspruchsdauern betroffenen Individuen (Programmgruppe) mit einer Kontrollgruppe von Arbeitslosen im Alter von 36-41 Jahren, für welche die Bezugsdauern vor und nach der Reform unverändert 12 Monate betrug, stellt somit eine Möglichkeit zur Identifikation des kausalen Effekts einer großzügigeren Arbeitslosenunterstützung dar.

Ein solcher Vergleich erfolgt in Arntz et al. (2008) auf der Basis einer 50 \%igen Stichprobe aller männlichen sozialversicherungspflichtigen Erwerbstätigen aus der BeschäftigtenHistorik des IAB. Während die durchschnittliche ALGAnspruchsdauer für die Kontrollgruppe vor und nach der Reform 12 Monate betrug, verkürzte sich die Anspruchsdauer für die Programmgruppe im Schnitt über alle betroffenen Individuen in unserer Stichprobe von 18,5 auf 12 Monate. Die Abschätzung des Effektes dieser Verkürzung der Anspruchsdauer, erfolgt mittels der so genannten kumulierten Inzidenzfunktion (KIF), d.h. der beobachteten Wahrscheinlichkeit bis zu einem Zeitpunkt $t$ die Arbeitslosigkeit zugunsten einer regionalen oder überregionalen Beschäftigung verlassen zu haben, indem die Veränderung der KIF für die Programmgruppe vor und nach der Reform der Veränderung der KIF für die Kontrollgruppe im selben Zeitraum gegenübergestellt wird. Unter der Annahme, dass beide Gruppen dieselben Veränderungen über die Zeit erfahren hätten, wenn für die Programmgruppe keine Kürzung der Anspruchsdauer vorgenommen worden wäre, kann die von der Kontrollgruppe abweichende Veränderung der KIF auf die Verkürzung der Anspruchsdauer zurückgeführt werden. ${ }^{8}$

Die Verkürzung der Anspruchsdauer zeigt für unqualifizierte Arbeitslose jedoch keine signifikante Wirkung auf deren räumliche Mobilität. Dieses Ergebnis ist jedoch nicht dahingehend zu interpretieren, dass die Arbeitslosenunterstützung keinen Einfluss hätte. Vielmehr ist der Effekt für diese Gruppe schwer nachzuweisen, da, wie oben erläutert, für diese Gruppe möglicherweise keine oder nur eine schwache Veränderung in deren Einkommenssituation stattfand. Für die vermutlich stärker betroffenen qualifizierten Arbeitslosen finden sich hingegen Hinweise darauf, dass eine großzügige Arbeitslosenunterstützung die Mobilitätsbereitschaft vor allem der Unverheirateten senkt, deren Mobilitätskosten im Vergleich zu Verheirateten vergleichweise niedrig ausfallen dürften. Während Verheiratete somit kaum

\footnotetext{
${ }^{8}$ Der hier verwendete nicht-parametrische Ansatz löst nicht das grundlegende Identifikationsproblem eines Verweildauermodells mit konkurrierenden Risiken, welches es nicht erlaubt die marginalen Verteilungen für die verschiedenen Übergangsmöglichkeiten zu identifizieren (vgl. Cox 1962; Tsiatis 1975). Veränderungen der KIF infolge der Reform können somit nur dann kausal auf die Reform zurückgeführt werden, wenn wir annehmen, dass die beiden konkurrierenden Risiken eines Abgangs in eine regionale und in eine überregionale $\mathrm{Be}$ schäftigung unabhängig voneinander sind. Der Schätzansatz hat jedoch den Vorteil, dass stattdessen Identifikationsprobleme, die in den Daten begründet sind, berücksichtigt werden können. Für Einzelheiten siehe Arntz et al. (2008).
} 
auf die verkürzte Anspruchsdauer mit einer erhöhten räumlichen Mobilität reagieren, sondern lediglich im lokalen Arbeitsmarkt schneller wieder eine Beschäftigung aufnehmen, hat die Arbeitslosenunterstützung bei den potenziell mobilen Unverheirateten auch einen Einfluss auf die Mobilitätsbereitschaft. Der Umfang der Arbeitslosenunterstützung hat somit zumindest auf Arbeitskräfte mit relativ niedrigen Mobilitätskosten einen mobilitätshemmenden Einfluss. Übertragen auf unqualifizierte Arbeitskräfte mit häufig sehr hohen Lohnersatzquoten ist daher davon auszugehen, dass die Arbeitslosenunterstützung besonders für diese Gruppen die Mobilitätsbereitschaft senkt.

\section{Diskussion und Ausblick}

Die skizzierten Forschungsarbeiten leisten einen Beitrag dazu, die Determinanten der Mobilität von Arbeitslosen in Deutschland näher zu beleuchten und mögliche institutionelle Gegebenheiten zu identifizieren, die zu einer niedrigen Mobilitätsbereitschaft, vor allem unter unqualifizierten Arbeitslosen, beitragen. Deren im Vergleich zu qualifizierten Arbeitslosen mangelnde Bereitschaft, auf ungünstige regionale Beschäftigungschancen durch Abwanderung zu reagieren, führt zu einer starken Abhängigkeit der Erwerbshistorie vom regionalen Arbeitsmarkt. Regionale Schocks schlagen sich unter Geringqualifizierten somit vermutlich stärker in den Partizipations- und Arbeitslosenzahlen nieder als für qualifizierte Arbeitskräfte mit einer höheren Mobilitätsbereitschaft.

Die Ursache dieser geringen Mobilitätsbereitschaft scheint jedoch nicht auf den Einfluss der aktiven Arbeitsmarktpolitik zurückzuführen zu sein. Vielmehr weisen die Forschungsergebnisse daraufhin, dass eine generöse Arbeitslosenunterstützung einen mobilitätshemmenden Einfluss ausübt. So konnte in zwei Studien gezeigt werden, dass eine verlängerte ALG-Anspruchsdauer die Wahrscheinlichkeit einer überregionalen Beschäftigung reduziert. Dieser Effekt ist jedoch nur für gut qualifizierte und unverheiratete Arbeitskräfte nachweisbar. Die fehlende bzw. schwache Reaktion unqualifizierter Arbeitskräfte auf eine veränderte ALG-Anspruchsdauer bedeutet jedoch nicht, dass die Arbeitslosenunterstützung für diese Gruppe keinen Einfluss hat, sondern ist vielmehr darauf zurückzuführen, dass eine Veränderung der ALG-Anspruchsdauer für diese Gruppe vielfach keine oder nur schwache Einkommensveränderungen mit sich bringt. Vor dem Hintergrund der Ergebnisse ist daher davon auszugehen, dass die dauerhaft hohen Lohnersatzquoten in Folge des Bezugs von Arbeitslosenhilfe und aufstockender Sozialhilfe eine hohe mobilitätshemmende Wirkung für einkommensschwache Gruppen haben.

Das Forschungsprojekt hat damit einen potenziellen Ansatzpunkt für die Politik identifiziert, die räumliche Mobi- lität in Deutschland zu heben, indem die mobilitätshemmende Wirkung einer generösen Ausgestaltung der Arbeitslosenunterstützung bei der Festsetzung von Lohnersatzquoten und Anspruchsdauern berücksichtigt wird. Gerade bei einkommensschwachen Arbeitslosen sind entsprechende Kürzungen jedoch weder politisch erwünscht noch mit dem Grundgesetz vereinbar. Zur Mobilisierung einkommensschwacher Arbeitskräfte könnten möglicherweise Programme dienen, die für eine dauerhafte Unterstützung eine Arbeitsaufnahme im sekundären Arbeitsmarkt verlangen. Die Wirkungen solcher Workfare-Programme auf die Mobilitätsbereitschaft sind bislang jedoch nicht erforscht.

Das Forschungsvorhaben konnte somit aufzeigen, dass institutionelle Rahmenbedingungen die individuelle Mobilitätsbereitschaft verändern können. Die Mobilitätslücke zwischen den Qualifikationsgruppen können die Ergebnisse jedoch nicht vollständig erklären. Dies hängt möglicherweise damit zusammen, dass die geringe Mobilität seitens unqualifizierter Arbeitskräfte auch darauf zurückzuführen ist, dass diese Qualifikationsgruppe besonders häufig mit unsicheren Beschäftigungsverhältnissen konfrontiert ist, so dass sich eine Investition in einen Umzug kaum auszahlt. Denn bei der Migrationsentscheidung spielen letztlich auch die Erwartungen über den zukünftigen Nutzen in einer potenziellen Zielregion eine Rolle, so dass Unsicherheiten über Einkommensmöglichkeiten eine entscheidende Rolle spielen können. Diese Hypothese steht auch mit weiteren Ergebnissen des Forschungsprojekts in Einklang, die darauf hindeuten, dass qualifizierte Arbeitskräfte vor allem in Regionen abwandern, die für sie aufgrund eines hohen Lohnniveaus gute Einkommen sichern, während unqualifizierte Personen Regionen bevorzugen, die über relativ stabile Beschäftigungsmöglichkeiten verfügen (vgl. Arntz 2010; Arntz et al. 2010). Eine gezielte Analyse des Zusammenhangs zwischen Beschäftigungsstabilität und räumlicher Mobilität steht jedoch noch aus.

Darüber hinaus sind die hier vorgestellten Haupterkenntnisse des Forschungsprojektes nur bedingt auf andere Länder übertragbar. So ist es denkbar, dass die generöse Arbeitslosenunterstuitzung die Mobilitätsbereitschaft von Arbeitslosen dominiert, so dass die aktive Arbeitsmarktpolitik in diesem institutionellen Rahmen kaum einen zusätzlichen Einfluss ausübt. In Ländern mit einer weniger generösen Arbeitslosenunterstützung könnte die aktive Arbeitsmarktpolitik somit trotz der hier vorgestellten Ergebnisse einen bedeutenderen Einfluss auf die Mobilitätsbereitschaft von Arbeitslosen ausüben. Um hier zu allgemeingültigeren Aussagen zu kommen, müssten zukünftige Forschungsvorhaben daher die Wechselwirkungen der verschiedenen Institutionen beispielsweise in länderübergreifenden Studien näher untersuchen.

Danksagung I would like to thank my Ph.D. supervisors Prof. Dr. Horst Entorf and Prof. Dr. Joachim Möller for all their support. I am 
also grateful for all the numerous comments at workshops and conferences from which this research project benefitted. Special thanks for productive collaborations go to my co-authors Ralf Wilke, Simon Lo, Terry Gregory and Florian Lehmer. Finally, I would like to thank Prof. Hübler and an anonymous referee for helpful comments on this paper.

\section{Literatur}

Arntz, M.: What attracts human capital? Understanding the skill composition of interregional job matches in Germany. Reg. Stud. 44, 423-441 (2010)

Arntz, M.: The geographical mobility of unemployed workers. ZEW discussion paper No. 05-34, Mannheim (2005) (updated version in Chapter 2 of Arntz, 2007)

Arntz, M., Gregory, T., Lehmer, F.: Unequal pay or unequal employment? What drives the self-selection of internal migrants in Germany. Mimeo (2010)

Arntz, M., Wilke, R.: Unemployment duration in Germany: individual and regional determinants of local job finding, migration and subsidized employment. Reg. Stud. 43, 43-61 (2009)

Arntz, M., Lo, S.M.S., Wilke, R.A.: Bounds analysis of competing risks: a nonparametric evaluation of the effect of unemployment benefits on migration in Germany. FDZ Methodenreport des IAB, 06/2008, Nürnberg (2008)

Bender, S., Haas, A., Klose, C.: The IAB employment subsample 1975-1995. Schmollers Jahrb. Wirtsch.- Soz.wiss. 120, 649-662 (2000)

Blanchard, O., Katz, L.: Regional evolutions. Brookings Pap. Econ. Act. 1, 1-75 (1992)

Braunerhjelm, P., Faini, R., Norman, V., Ruane, F., Seabright, P.: Integration and the Regions of Europe: How the Right Policies Can Prevent Polarization. CEPR, London (2000)

Choy, W.K., Maré, D., Mawson, P.: Modelling regional labour market adjustment in New Zealand. New Zealand Treasury working paper No. 02-01, New Zealand (2002)

Cox, D.R.: Renewal Theory. Methuen, London (1962)

Damm, A.P., Rosholm, M.: Employment effects of dispersal policies on refugee immigrants. Part I. Theory. IZA discussion paper No. 924, Bonn (2003)

Debelle, G., Vickery, J.: Labour market adjustment: evidence on interstate labour market mobility. Aust. Econ. Rev. 32, 249-263 (1999)

Decressin, J.W., Fatàs, A.: Regional labor market dynamics in Europe. Eur. Econ. Rev. 39, 1627-1655 (1995)

Faini, R.: European migrants: an endangered species? In: Baldwin, R.E. et al. (Hrsg..) Market Integration, Regionalism and the Global Economy, S. 228-251. Cambridge University Press, Cambridge, (1999)

Fallick, B.C.: Job security and job search in more than one labor market. Econ. Inq. 3, 742-745 (1992)

Frederiksson, P., Johansson, P.: Employment, mobility, and active labor market programs. IFAU working papers 2003-3, Uppsala (2003)

Hassler, J., Rodriguez Mora, J.V., Storesletten, K., Zilibotti, F.: A positive theory of geographic mobility and social insurance. Int. Econ. Rev. 46, 263-303 (2005)
Herzog, H.W., Schlottmann, A.M.: Labor force mobility in the United States: migration, unemployment and remigration. Int. Reg. Sci. Rev. 9, 43-58 (1984)

Herzog, H.W., Schlottmann, A.M., Boehm, T.P.: Migration as spatial job-search: a survey of empirical findings. Reg. Stud. 27, 327-340 (1993)

Jackman, R., Savouri, S.: Regional migration in Britain: an analysis of Gross flows using NHS central register data. Econ. J. (Lond.) 102, 1433-1450 (1992)

Jacobebbinghaus, Seth, S.: The German integrated employment biographies sample IEBS. Schmollers Jahrb. Wintsch.-Soc.wiss. 127, 335-342 (2007)

Lindgren, U., Westerlund, O.: Labour market programmes and geographical mobility: migration and commuting among programme participants and openly unemployed. IFAU working paper No. 6, Uppsala (2003)

McCall, B.P., McCall, J.J.: A sequential study of migration and job search. J. Labor Econ. 5, 452-476 (1987)

Möller, J.: Empirische Analyse der Regionalentwicklung. In: Gahlen, B., Hesse, H., Ramser, H.J. (Hrsg.) Standort und Region, Bd. 24, S. 197-230. Mohr, Tübingen (1995)

Nahuis, R., Parikh, A.: Factor mobility and regional disparities: East, West, home's best? CPB discussion paper No. 004, University of East Anglia (2002)

OECD: OECD Employment Outlook 2005. Paris (2005)

Pissarides, C.A., Wadsworth, J.: Unemployment and the inter-regional mobility of labour. Econ. J. (Lond.) 99, 739-755 (1989)

Puhani, P.A.: Labour mobility - an adjustment mechanism in Euroland? Empirical evidence for Western Germany, France and Italy. Ger. Econ. Rev. 2, 127-140 (2001)

Shields, G.M., Shields, M.P.: The emergence of migration theory and a suggested new direction. J. Econ. Surv. 3, 277-304 (1989)

Sjaastad, L.A.: The costs and returns of human migration. J. Polit. Econ. 70, 80-93 (1962)

Thomas, J.M.: The role of selective job search in UK unemployment. Econ. J. (Lond.) 108, 646-664 (1998)

Tsiatis, A.: A nonidentifiability aspect of the problem of competing risks. Proc. Natl. Acad. Sci. USA 72, 20-22 (1975)

Westerlund, O.: Internal migration in Sweden: the effects of mobility grants and regional labour market conditions. Labour 12, 363-388 (1998)

Westerlund, O.: Employment opportunities, wages and interregional migration in Sweden 1970-1989. J. Reg. Sci. 37, 55-73 (1997)

J. Prof. Dr. Melanie Arntz is a senior researcher at the Centre for European Economic Research (ZEW Mannheim) and the deputy head of the ZEW Research Department "Labour Markets, Human Resources and Social Policy". She studied geography at the University of Bonn and at the University of Minnesota and finished her doctoral thesis regarding "The Geographic Mobility of Heterogenous Labour in Germany" at TU Darmstadt in 2007. In 2009, she joined the faculty of the University of Heidelberg as an assistant professor for "Labour Markets". Her research focuses on the dynamics of individual labour market careers, regional mobility and regional labour markets. 